\title{
Why PHR when EHR is the record of the future?
}

\author{
Dr. G. D. Mogli Ph.D., FHRIM (UK), FAHIMA (USA) \\ Senior. Consultant eHealth Management, HEARTCOM INC. (USA) \\ E-Mail address: gdmogli@yahoo.com
}

Sri Lanka Journal of Bio-Medical informatics 2011:2(2):75-83

DOI: http://dx.doi.org/10.4038/sljbmi.v2i2.2246

\begin{abstract}
The 20th century has revolutionised the medical records system from outpatient cards to comprehensive unit records due to its important role played in effective healthcare delivery. The unit record system served almost the latter half of the 20th century. The theme of this paper is "Why PHR when EHR is the record of the future". Examination of medical record system practiced in the last century will enlighten as to what type of medical records are required for the 21 st century and beyond so that everyone will get swift, safe and good quality care at affordable cost.

The physician needs comprehensive information to provide effective care, depending on the patient's record that is not easily available as patient care information is scattered or disintegrated. A patient gets treatment at different health organisations, treats himself or uses other services such as herbal, unani, homeopathy or ayurveda. Despite the fact EHR is proved to be a great potential and the 'record of future', implementation difficulties due to lack of application of international standards like HL7, SNOMED-CT, LOINC, ICD, DIOCOM, NCPDP etc., are required to be fully interoperable.

The PHR is defined as 'a Health Passbook containing the identification data of a Person', is a lifelong electronic, universally available document, initiated at the time of birth, containing, entire information such as episodic, hospitalisation, self medications and other habits including significant events, advance directives of living wills, organ donor authorisation, usually not available to care providers. This Health Passbook should have the patient identification data, health summary, child development, immunisation, self care/treatments, medications, investigations, hospitalisation, obstetric \& gynecology, therapy, chronic disease (old age), and dental.

Effective EHR at health institution level and PHR at personal level linking together and complementing each other will add value for accomplishing complete 360 degree information that will help provide comprehensive continuity of care at the right time, right place and at right cost. This system will prevent duplication of investigations, medications, delay in care, check on risk and cost.
\end{abstract}

Keywords - EHR; PHR; medical record system

\section{Healthcare system}

Health and records run parallel to each other as the health condition is measured via medical records and any national rank is calculated with the health status of the population. Thus, it is mandatory to ensure that everyone should have reasonably good health. To achieve this, we need to understand the system.

Any sick or injured person usually visits one of the following places: physician's office, primary healthcare centre, emergency department or hospital. It could be a minor health issue with low risk or a major medical or surgical emergency with a high risk condition. Medical record maintains health accounts of everyone who visit a health institution. The care providers range 
from medical specialist to nurses and other paramedical teams along with the allied health service providers who are directly or indirectly involved.

\section{Medical Record is the mother of primary health information}

Every person visiting a physician's office or hospital need not be a patient, it could be a regular visit. Reasons can vary from a well baby clinic visit to an immunisation or a health promo, or a medical check - up or a consultation. If the visits are documented on par with international standards, the information will be of great value to many. Particularly to patients, doctors, hospitals, legal, insurance, teaching and research teams and other national and international organisations. This record is the original document and the mother of primary health information from where secondary data is produced. If this can be adhered universally, health status will be far better at a global level.

\section{Physicians depend on patient health information}

Although, the medical fraternity is well qualified to deal with all types of cases, they still need to know the background information of a patient such as medical history, family history, general and dietary habits, living conditions. Ecological and social conditions reflect a clear picture to establish a correct diagnosis to render appropriate treatment. A healthcare provider will require information such as age, gender, occupation, social and economical background etc. He will then elicit information such as the type of treatment / medication being revived, blood group, allergies, any congenital anomalies etc. All this information will be recorded to provide a comprehensive report of a patient to facilitate treatment plan.

\section{Problem of maintaining integrated records}

Maintenance of integrated health information pertaining to a single person has become a formidable task despite excellent medical record systems being practiced in most technologically advanced countries.

This means, the patient information is scattered and disintegrated in different health organisations. This happens not only because of patients' extensive movement, but even because within the same institution, a patient visits different clinics or specialties, each specialist doctor is only interested in a given subject. This leads to all information not been available to everyone within the same institution.

One patient is divided into different parts for the purpose of recording information. Ideally, this is expected to give a clear picture but instead, leads to duplication of services, efforts, expertise leading to confusion. Delays add to patient's risk, incurring additional expenditure to already limited funds available for health care.

\section{Incomplete information}

With the best of efforts of unifying the unit record system within a health institution, whatever healthcare rendered is not completely accessible. Even patient records are too many, and 
scattered in different files, different pages, different areas, and different order. It is extremely difficult to a busy physician to scan a variety of specialised papers to arrive at a conclusion. Besides, patients receive care in a variety of places and also at times, resort to self-medication or self-recommended care, and also may get herbal, homeopathic, unani or ayurveda treatment. This information is generally not available and capturing all these will be hard and time consuming.

There is a dire need to improve the entire information record system which will help a care provider to come to a quick decision. This will lead to safe and fast care giving, minimising duplication of efforts, and resources. The best is Personal Health Record.

\section{Personal Health Record}

The PHR is a life long record initiating from birth, through neonatal, infancy, childhood, adult, and old age till death. The PHR will be maintained by the patient or the guardian (in the case of a child or an unsound person). This will be carried wherever he moves so that it can meet any necessity of health issues anywhere, at any time. I would like to go into details as to whether the PHR is a solution or is it only an extension to existing system of maintenance of medical records including EHR.

\section{Materials and methods}

Examination of medical record system practiced in the last century will enlighten as to what type of medical records are required for the 21 st century and beyond so that everyone will get swift, safe and good quality care at an affordable cost. The healthcare delivery system and health records which are a reflection of care provided, need to be thoroughly examined to find the right solution to the problem. As a first step, let us understand the evolution of medical record system that might throw some light. As regards the methods used to understand the hypothesis, the past and present medical practice and medical record system to be evaluated to have a clear picture and the need for PHR will be decided.

\section{Historical background}

The history of the medical record parallels the history of medicine. Primitive records existed since 5000B.C., changing circumstances are responsible for stimulating the evolution of improved methods. The greatest improvements in medical record services were derived from the hospital standardisation movement in 1918. By the onset of the 20th Century, a vigorous and widely accepted move of patient records system became apparent in the United Kingdom. After World War I, physicians used case records to study the results of new techniques in medicine and surgery. During World War II, standardised documentation maintained through the air raid casualty and service records as part of the Emergency Medical Service was introduced in many countries. The evolutionary changes in healthcare in Western countries greatly contributed to the scientific medical records to improve organisation of medical record services and medical record staff. 


\section{Maintenance of medical records}

The 20th century has seen a significant growth in medical records while initially outpatient chit (card) kept by the patient was used in many clinics and physicians' offices. Due to compulsion for improvement, the comprehensive unit record system was introduced. The unit record system concept is that each patient should have only one case record folder into which all documents relating to his history of medical care are placed. This system offers a complete history to all clinicians involved with the care and treatment of the patient. This system was used in the second half of the 20th century and with the dawn of Information Technology (IT), the electronic medical record with different terminology evolved to be finally termed as the Electronic Health Record (EHR).

\section{Electronic Health Record (EHR)}

An EHR refers to a patient's longitudinal medical record in digital format. EHR systems coordinate the storage and retrieval of records with the aid of computers. EHRs are usually accessed on a computer, often over a network. It may be made up of Electronic Medical Records (EMRs) from many locations and/or sources. A variety of types of healthcare-related information may be stored and accessed in this way. EHR systems can reduce medical errors, increase physician efficiency and reduce costs, as well as promote standardisation of care.

The EHR is definitely far superior to all the above systems, and has remarkable benefits in implementing the system. The biggest advantage of this system is that a comprehensive record is instantly accessible to all the authorised care providers from different stations and all related documents can be seen on online. Information is secured and portable via internet.

\section{Results}

Having learned various systems that were being practiced in the 20th century now indicate that there has been significant transformation in the improvement of patient record system due to dire need. The EHR is gradually revolutionising the healthcare delivery system. It will be a key achievement for all countries to implement a standardised, interoperable electronic system.

\section{Need for Personal Health Record (PHR)}

PHR implementation has become necessary because of not having a composite picture of a patient. This drawback delays prompt care, increases errors and risk, leads to duplication of efforts and resources which lead to high costs. Researchers observe that PHR can be carried by the patient wherever he goes. If he needs any medical assistance, he can readily produce the PHR thereby facilitating the decisions and the service of the next care giver. It can also contain living wills and advance directives to organ donor authorisation. The implementation of the mechanism appears simple, but requires extensive study. 


\section{Discussion}

Records maintained by a physician's office or a hospital are focused on legal and financial concerns, whereas EHR and PHR is more patient/ personal health oriented. The PHR will have a complete integrated story relating to a patient's health. This will encompass self initiated care. This record will act as a gap filling document between healthcare institutions and self medication. Patient record is divided in to two; where a picture of the human body is on one side while the other side gives a picture of common problems with timelines and habits etc. of the patient. All this will add to the list of comprehensive information in order to understand health trends. This will have a significant impact on providing data for research and will help in improving general lifestyle of a patient.

\section{What is PHRs}

The "PHR is a health passbook containing the identification data of a person. It is a lifelong electronic, universally available document, initiated at the time of birth. The PHR is maintained by parents/guardian till the child become of age. Each individual owns and manages the information and it will be placed in a secure and private environment of his choice.

\section{Beginning of PHR}

Those born prior to implementing PHR will need to request a copy of their current health record from their current healthcare providers. A patient needs to obtain an 'authoriation for the release of information' form from their respective health care providers. At least, past operative reports, discharge summaries, and significant tests from any hospital visit could be collected and placed in PHR.

The PHR is a simple health passbook which can be maintained on your home computer or via a reputed web site. When traveling, the patient can carry a portable device which will contain the PHR. Patients with special medical conditions such as diabetes, hypertension, or heart conditions should consider wearing an alerting device. If your healthcare provider maintains a web site where you can maintain your own health records, follow their instruction. Review the notice of privacy practices provided to you by your healthcare provider.

\section{Purposes of PHR}

Maintaining one's own personal health record is one of the best ways to have constant access to his/her health information over the course of a lifetime. Whether one changes physicians or physician relocates or retire, by keeping own personal health record, the person and his family will have vital information at their disposal at any time; traveling or otherwise. With this information one can provide information to new caregivers, and discuss all aspects of his/her health issues.

\section{How PHR can be implemented}


In fact, PHR is an extension of EHR. As we have observed medical records whether manual or electronic are being maintained mostly by healthcare providers and organisations. Exception to this, is that child heath, maternal and chronic disease records are allowed to be carried by patients. However, since travel has become common lately, it has proved that health records maintained by healthcare organisations may not fulfill the comprehensive or emergency needs as the case may be. Besides, many of us use personal judgment in the case of minor ailments. At times, a patient might even opt for alternate medicines such as homeopathy, unani, herbal or ayurveda etc.

\section{Problems of EHR implementation}

However, the EHR has proved to be a great potential and the 'record' of the future, it also experiences implementation difficulties, due to the development of softwares tailor made to suit certain health institutions which hinders the exchange of information. This is also a hurdle and is being tackled by applying various international standards like HL7, SNOMED-CT, LOINC, ICD, DIOCOM, NCPDP etc., to be fully interoperable.

\section{General attitude toward direct patient access to EHR}

A survey revealed that most patients believe that having access to their EHR will help them improve their healthcare decision making process. Most preferred the internet as the medium of record, but preference varied with age, with older patients preferring paper. Clinicians preferred online patient care tools.

\section{Starting your PHR}

The EHR is maintained by the health organisation for the care of patients; and administrative, legal, medical education and research purposes. However, when patient receives treatment, the information has to be shared with PHR. Similarly, when a patient is in the care of a different organisation, EHR has to be updated accordingly.

The PHR passbook should have the following information:

- Patient Identification Data

- Health summary

- Child development information

- Immunisation records

- Self care/treatment records

- Medications

- Investigations

- Hospitaliation records

- Obstetric and gynecology

- Therapy

- Chronic disease (old age)

- Dental

Patient ID format 
Contains three parts; part I contains personal data, viz. name, age (date of birth), gender, social security number, birth identification marks, emergency contact address, insurance details. Part II contains significant health issues, eg. allergies etc., Part III contains other habits such as food, alcoholic, smoking, addictions etc.

\section{Health summary}

This is a comprehensive format, providing for the record of chronological data. Whenever, any health issue occurs, patient should record the necessary data. If he attended outpatient clinic, starting and ending date of episode, type of service received, investigations, medications and results should be recorded. Similarly, information and data such as details of visits to the emergency room, details of hospitalisation records and diagnosis data should be compiled. In the case of self medication or self recommended treatment, such details also should be recorded in the appropriate format.

This record is a duplicate of which is in the physician's office or OPD clinic, or ER. The health institutions will be maintaining records as usual for administrative, legal and other purposes. However, there will be a note in the PHR with date of admission and discharge and name of institution and contact address. In case of emergency, the information should be easily accessible. Similarly, other information could also be accessible. Physician's office and OP clinic data is already available in the PHR. Information regarding alternate medication is also recorded.

This is a growth chart including weigh and height. A document for children from age 0-5 years or 0-14; years where all childhood health issues and other well baby clinic data are recorded. Any significant information such as child psychology information is also recorded.

\section{Immunisations}

This document is mainly for children but can also be used for adults. This will indicate the due dates for other immunsations.

\section{Self-care}

This record is unique, because it is available only with the PHR and not found in the allopathic healthcare organisations.

\section{Medication}

This will give a chronological account of medications administered, and highlight on current medications used. E-prescription, refills, and address of the pharmacies.

\section{Investigations}

Chronological account of investigations carried out will be available. With the facility to have a cumulative picture of different tests with dates will give the comparison that will greatly facilitate the care giver. 


\section{Hospitalisation record}

This will give a chronological account of all admissions and discharges. A hyperlink which will be directed to the domain of the physician or institution will be provided giving access of references of any data.

\section{Obstetrics and gynecology}

This document is for female patients starting from child bearing age onwards.

\section{Therapy}

Different types of therapies, such as physical, occupational, speech, optometric refractions, radio therapy etc.

\section{Chronic disease record}

This record will indicate medical data of a patient suffering from any chronic disease for example, diabetics, hypertension, cancer, arthritis etc.

\section{Dental}

This means all information regarding dental health from childhood to old age, dealing with dentures, cosmetics to extractions etc.

\section{Conclusion}

Effective EHR at health institution level and PHR at personal level linking together and complementing each other will add value to achieving a comprehensive set of information that will help provide a comprehensive continuity of care, at the right time, right place and right cost. This system will not only facilitate in providing the best possible and timely healthcare, but also prevent duplication of investigations, medications, delay in care, risk and reduce cost. Standardising of PHR information is a must as the type of information it contains is a continuity of care as patient focused record allows practitioners from different settings and disciplines to share information and that allows the patient to carry this information with him or her upon referral, transfer, or discharge. The PHR can help with better understanding of the patients' role as a partner with their

provider.

\section{References}

1. AHIMA e-HIM Personal Health Record Work Group "The Role of the Personal Health Record in the EHR." Journal of AHIMA 76, no.7 (July-August 2005): 64A-D.

2. Harold P. Lehmann and others; Aspect s of Electronic Health Record Systems Personal 
Health Record (PHR), pp50-51,100, 153. Available from: http://healthinsurance.about.com/od/glossary/g/phr.htm (Accessed on 10 December 2011)

3. Margaret K. Amataykul "Electronic Health Records," PHR(2004) pp 11, 206.

4. "Medical Record Consultant's Role in Implementing Electronic Health Record" published in Proceedings of 15th International Health Record Congress, held at Seoul, South Korea, 26-31 May, 2007.

5. Mogli, G.D. "Managing Medical Records” Channel Publications, USA 1996, pp 6-7

6. "Standardization of Paperless Health Record" published in Journal of IHRIM (UK); 1995;36(1):10-12 